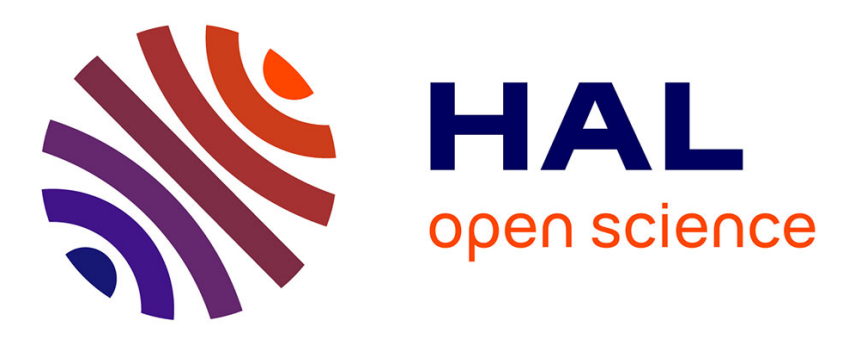

\title{
A Simple Compensation Method for the Accurate Measurement of Magnetic Losses With a Single Strip Tester
}

\author{
Olivier de La Barrière, C Ragusa, M Khan, C Appino, F Fiorillo, F \\ Mazaleyrat
}

\section{To cite this version:}

Olivier de La Barrière, C Ragusa, M Khan, C Appino, F Fiorillo, et al.. A Simple Compensation Method for the Accurate Measurement of Magnetic Losses With a Single Strip Tester. IEEE Transactions on Magnetics, 2016, 52 (5), 10.1109/TMAG.2016.2527829 . hal-01327663

\section{HAL Id: hal-01327663 https://hal.science/hal-01327663}

Submitted on 6 Jun 2016

HAL is a multi-disciplinary open access archive for the deposit and dissemination of scientific research documents, whether they are published or not. The documents may come from teaching and research institutions in France or abroad, or from public or private research centers.
L'archive ouverte pluridisciplinaire HAL, est destinée au dépôt et à la diffusion de documents scientifiques de niveau recherche, publiés ou non, émanant des établissements d'enseignement et de recherche français ou étrangers, des laboratoires publics ou privés. 


\title{
A simple compensation method for the accurate measurement of magnetic losses with a single strip tester
}

\author{
O. de la Barrière ${ }^{1}$, C. Ragusa ${ }^{2}$, M. Khan ${ }^{2}$, C. Appino ${ }^{3}$, F. Fiorillo ${ }^{3}$, F. Mazaleyrat ${ }^{1}$ \\ ${ }^{1}$ SATIE, CNRS, UniverSud, 61 av du President Wilson, F-94230 Cachan, France \\ ${ }^{2}$ Dipart. Energia, Politecnico di Torino, C.so Duca degli Abruzzi 24, 10129 Torino, Italy \\ ${ }^{3}$ Nanoscience and Materials Division, Istituto Nazionale di Ricerca Metrologica (INRIM), Torino, Italy
}

\begin{abstract}
We present a new method for the accurate characterization of soft magnetic sheets using a permeameter based on the precise compensation of the magnetomotive force (MMF) drop in the flux-closing yoke. It has been developed in order to overcome the systematic uncertainty affecting the value of the magnetic fieldstrength in single sheet testers when obtained, according to the standards, through the measurement of the magnetizing current. This phenomenon is more critical for high permeability materials, because of the reduced MMF drop across the sample. While additional sensors and auxiliary windings have been proposed in the literature, a novel approach is demonstrated here, based on the use of the permeameter upper half yoke as the MMF drop sensor and of an auxiliary winding on the lower half yoke, implementing compensation. This solution, dispensing one from dealing with the usually small signal levels of the conventional MMF drop sensors (e.g. Chattock coils), provides best results with the introduction of wedge-shaped magnetic poles, in order to accurately define the magnetic path length. The method is validated by measurements of power loss, apparent power, and hysteresis cycles on non-oriented and grain-oriented Fe-Si steel sheets, which are compared with local measurements performed on the same samples using $\boldsymbol{H}$-coil and $\boldsymbol{B}$-coil across a uniformly magnetized region.
\end{abstract}

Index Terms-Magnetic loss, hysteresis cycle, compensated permeameter, apparent power.

Tis I. INTRODUCTION
HE PROPER design of electrical machines an67 electromagnetic devices requires an accurate prediction of 8 iron loss. Indeed, in modern embedded applications such a39 hybrid or electric vehicles [1][2], the best compromise must b $\$ 0$ found between the machine efficiency [3][4] and torqu 1 density [5]. But any predictive model starts from a prope 2 material characterization [6][7]. The standard testing3 technique of soft magnetic steel sheets is based on the use of 4 the Epstein test frame [8]. It shows good reproducibility, a $\$ 4$ demonstrated by inter-laboratory comparisons of power los 46 and apparent power measurements [9]. However, this method47 besides requiring tedious preparation of the samples, i $\$ 8$ inevitably affected by appreciable systematic deviations (up t $\$ 9$ about $10 \%$ at high inductions) from the true values of thø0 measured quantities, as obtained, for example, by accurat51 measurements using $H$-coils [9][10][11]. But the $H$-co $\$ 2$ method requires the integration of low-level signals and i\$3 hardly acceptable in the industrial practice. Increasing interes 4 is therefore attached, at present time, to the Single Shee 5 Testing (SST) method, applied according to the pertaining IE 56 Standard [12][13], because of the convenient use of wides7 lamination samples. The STT method does not require stres 58 relief of samples upon cutting, can be directly applied to the ${ }^{9}$ domain-refined high-grade grain-oriented materials, and shows good reproducibility of measurements [9] [14 Consequently, there is demand by industry for including SST reference values in the material specification standards. With 64 the SST arrangement, where the sheet sample is inserted in 85 double-C laminated yoke, the magnetic fieldstrength i 86 calculated using the measured magnetizing current and 87 defined magnetic path length $\left(l_{\mathrm{m}}=0.45 \mathrm{~m}\right)$ is adopted. A main8 problem here is that the magnetomotive force (MMF) drop is9 the flux-closing yokes may not be negligible with respect to the one across the sample, especially with high-permeability materials. This can lead to overestimated magnetic field values. At the same time, the yoke itself can provide a certain contribution to the measured loss, depending on its manufacture and the possible existence of interlaminar eddy currents at the pole faces [15].

One way to overcome this difficulty is, as discussed in [16], one of compensating the drop of the MMF in the yokes of the permeameter by an auxiliary magnetizing winding. This is driven by a Chattock coil, placed over the measuring sample region of length $l_{\mathrm{m}}$ and a very high-gain amplifier, implementing a feedback control on the auxiliary current, such as to compensate the MMF drop outside the length $l_{\mathrm{m}}$ [16]. Like all the $H$-coil measurements, this method has a weak point in the necessity of handling small signal levels.

In this paper, we consider a single strip double- $\mathrm{C}$ yoke permeameter, applied to annealed Epstein samples, where a new compensation method, simpler and more effective than previous literature solutions [16], is implemented. Epstein strip samples are used in the present experiments for practical convenience, but the method could be easily adapted to standard SST permeameters. The idea is one of using the upper half of the yoke as a zero MMF indicator and the lower half for accommodating the compensation circuit. It is a simple measuring arrangement, where the signal to be controlled is relatively large and much easier to handle than the weak and noise-prone signal generated by a Chattock coil. As a further advantage, there is no geometrical discontinuity in the magnetizing winding, as required instead, with ensuing inhomogeneity of the applied field, by the insertion of the Chattock coil [16]. The method is validated by comparison with accurate measurements performed upon a relatively restricted median region of the strip sample by the $H$-coil method. Non-oriented (NO) and high-permeability grainoriented (GO) Fe-Si samples have been tested, with very good 
Fig 1 - Sample (Epstein strip), with enwrapping $B$-coil and tangential $H$ - coil (3D view and front view)

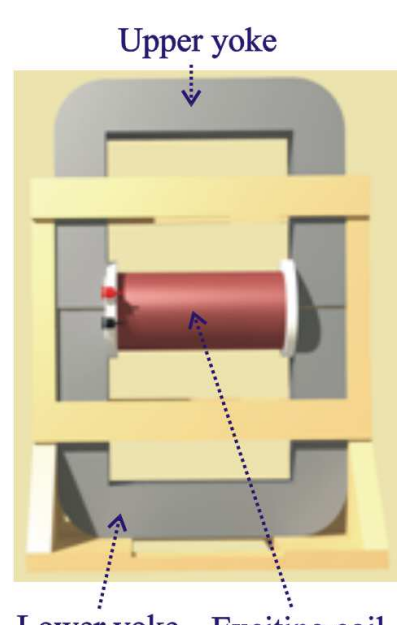

Lower yoke Exciting coil

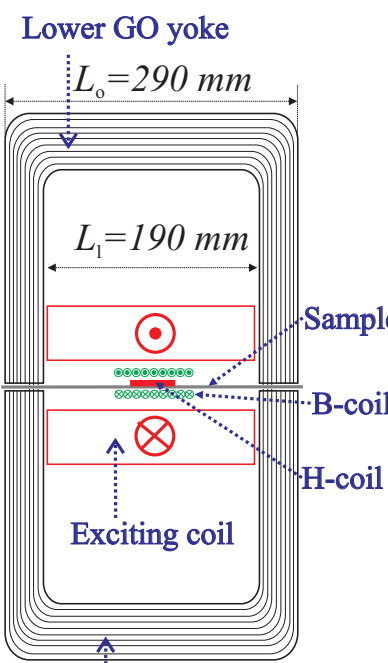

Upper GO yoke
Fig. 2 - a) 3D view of the permeameter. b) 2D view of magnetizer, excitihg 8 circuit, and measuring coils.

agreement between the results obtained with the magnetizing0 current and those obtained by localized $H$ and $B$ coils.

In this section we describe the geometry of the system and 105

\section{A. System geometry and magnetic path length}

The sample is a conventional annealed Epstein strip. The $\mathrm{mm}$ long pickup coil placed at the center of the strip. Inside the $B$-coil, a many-turn calibrated flat $H$-coil $(1 \mathrm{~mm}$ thick turn-area $2.25 \cdot 10^{-2} \mathrm{~m}^{2}$ ) of same length is placed upon the

Fig. 1). The $H$-coil provides, after integration of the measured voltage, the tangential field upon the measuring 112 area. Since in the measuring region the applied field and the are verified to be highly uniform, tangential field oss is thus identified with the true loss figure of the material, hat is, the reference quantity for the results obtained with the

The developed permeameter, shown in Fig. 2, consists of 30 double-C laminated yoke of $50 \mathrm{~mm} \times 50 \mathrm{~mm}$ cross-sectiona 1 area, made of $0.30 \mathrm{~mm}$ thick high-permeability GO strips. $1 \$ 2$ uniformly wound magnetizing solenoid covers the distance 133 $=190 \mathrm{~mm}$ between the pole faces of the yoke. It is endow $\$ 94$ with series connected additional narrow windings at its ends35 by which maximum uniformity of the applied field across the whole distance $L_{\mathrm{i}}$ is obtained ([11], p. 109).

According to Ampère's law, the magnetomotive force $N I$ generated by a current $I$ flowing into the $N$-turn solenoid is related to the magnetic field $H$ along a closed path $L$ by the equation

$$
N \cdot I=\oint_{L} H \cdot \mathrm{d} l
$$

If a method is found by which the drop of the MMF in the flux-closing yoke is made either negligible or fully compensated, (1) simply becomes $N \cdot I=H \cdot L_{\mathrm{s}}$, where $L_{\mathrm{s}}$ is the length of the mean magnetic flux path in the sample. $L_{\mathrm{s}}$ has value intermediate between $L_{\mathrm{i}}$, the distance between the pole faces, and $L_{0}$, the width of the permeameter (Fig. 2b), as the obvious result of flux channeling from the sample into the yokes (see Fig. 3a). It is actually not well defined and it depends on the magnetization level in the sheet sample. It will appreciably change on approaching the material saturation.

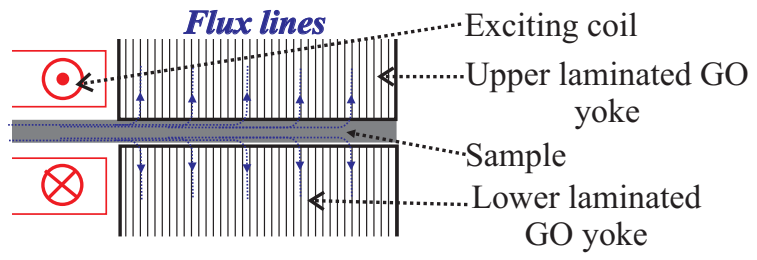

(a) Standard configuration.

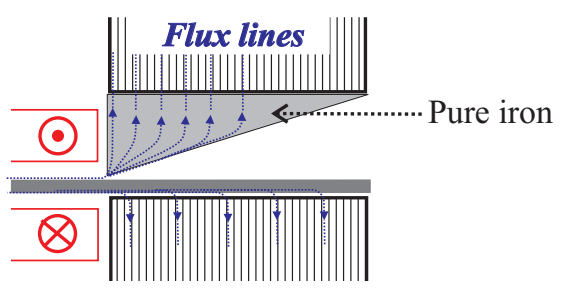

(b) Modified configuration.

Fig. 3 - Defining the magnetic flux path length in the sample.
We wish to impose a flux path such that the length $L_{\mathrm{s}}$ coincides with the distance between the pole faces $L_{\mathrm{i}}$. To this end, the following modification of the upper yoke is proposed. Two identical wedge-shaped pure iron poles are placed beneath the two limbs of the upper yoke, as shown in Fig. 3b and Fig. 5, with the contact lines between the sheet surface and the poles placed exactly at the distance $L_{\mathrm{i}}$. As discussed in the following Section, this is expected to force, under the action of a feedback system employing auxiliary windings on the yokes, the flux path length in the sample to be equal to $L_{i}$. Under these conditions, the magnetic field $H$ in the sample will be obtained according to the relation

$$
N \cdot I=H \cdot L_{i} .
$$

independent of the way the flux lines enter the lower yoke.

\section{B. The principle of MMF compensation.}

The idea here developed follows to some extent from the method using a Chattock coil sensor to cancel the MMF on a known length, as described in [16]. Here we take the yoke itself as a zero MMF indicator. A few-turn secondary coil wound around the upper yoke provides the derivative of the magnetic flux $\varphi_{1}$ flowing in it. According to the simplified reluctance description of the system shown in Fig. 4, where 
137 the reluctance of the sample is $R_{\mathrm{S}}$, the reluctance of the uppler4

and lower yokes is $R_{\mathrm{Y}}$, and that of the wedge-shaped pole 1 i 85 the sample is proportional to the flux $\varphi_{1}$. Since there is 987 MMF source in the upper yoke, $E$ will be reduced to zero $\$ \$ 8$ canceling the flux $\varphi_{1}$, that is, bringing to zero the voltaq89 correspondingly induced in the secondary coil. This can $\mathrm{P}_{0}$ accomplished by adding a compensation winding on the lower 1 yoke and controlling it in such a way that the corresponding $y_{2} 2$ generated MMF $N_{\mathrm{c}} I_{\mathrm{c}}$ leads to the condition $E=0$. The contro $\}_{3}$ loop is schematically shown in Fig. 5. An analog control card (PID controller) keeps the voltage $v_{1} \propto \mathrm{d} \varphi_{1} / \mathrm{d} t$ equal to zero, by 4 properly supplying, via a high-gain linear amplifier, th $\$ 5$ compensation winding on the lower yoke. Consequently, $\mathbf{a} \not 6$ the flux $\varphi_{\mathrm{S}}$ in the sample is made to entirely flow in the lower yoke. $R_{\mathrm{P}}$, the MMF drop $E$ pertaining to the magnetic circuit outsid8 86

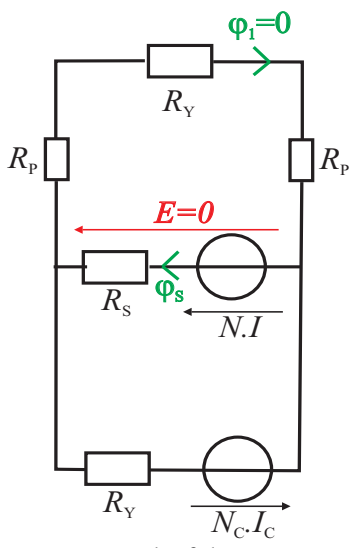

Fig. 4. Reluctance network of the compensated permeameter.

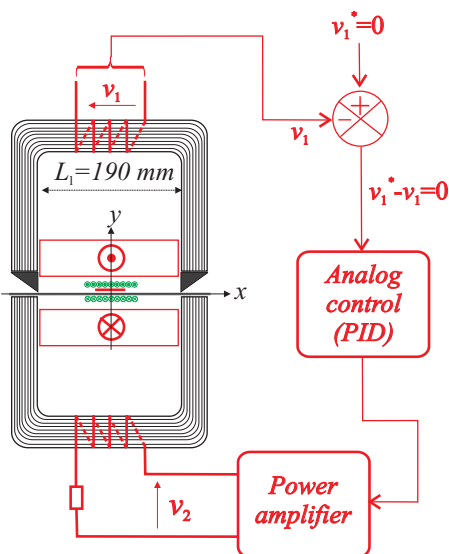

Fig. 5. Compensated permeameter and control circuit.

This compensation method is simple and sensitive, sincel 186

154 does not require any specific MMF sensor and a high signdi87 to-noise ratio is ensured by the high permeability of the G.88 aminations employed in the yokes. The voltage $v_{1}$ is, fp 89 example, always much larger than the one achievable by ${ }_{190}$ Chattock coil, and its control around the zero value is 1 correspondingly easier and more precise. To be remarked that imperfect contact between the wedge shaped poles and the sample, which can be lumped in the pole reluctance $R_{\mathrm{P}}$, has 194 little detrimental effect on the permeameter performance 194 because the flux in the upper yoke is made to vanish.

\section{EXPERIMENTAL RESULTS}

The novel compensated permeameter has been tested on nonoriented Fe-(3 wt\%)Si sheets (thickness $0.35 \mathrm{~mm}$ ) and high-permeability $(\mathrm{HiB})$ grain-oriented sheets (thickness $0.28 \mathrm{~mm}$ ). Energy loss, apparent power, and hysteresis loops were measured under sinusoidal induction waveform at $f=$ $100 \mathrm{~Hz}$ and peak polarization values $0.2 \mathrm{~T} \leq J_{\mathrm{p}} \leq 1.5 \mathrm{~T}$. The value of the magnetic field $H$ was obtained both by measuring the magnetizing current and by integrating the signal induced in the $H$-coil placed at the center of the Epstein strip.

\section{A. Non-oriented Fe-Si sheets}

Fig. 6 shows the measured energy loss vs. peak polarization obtained through the $H$-coil and current methods.

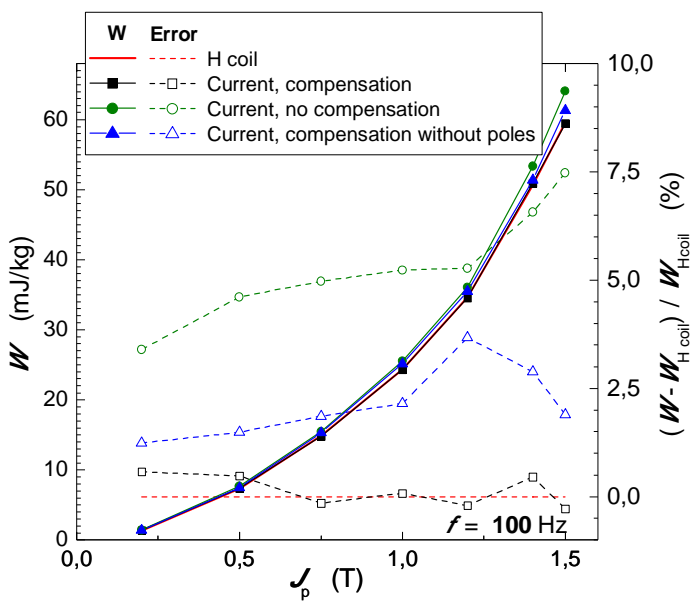

Fig. 6. NO Fe-Si sheets. Comparison of the energy loss measured by the $H$ coil method and the current measurement method. The permeameter can be compensated as described above, non-compensated (the compensation circuit is not switched on), or compensated without the iron poles. The dashed lines provide the deviations of the loss figures measured with the current method with respect to the $H$-coil method.

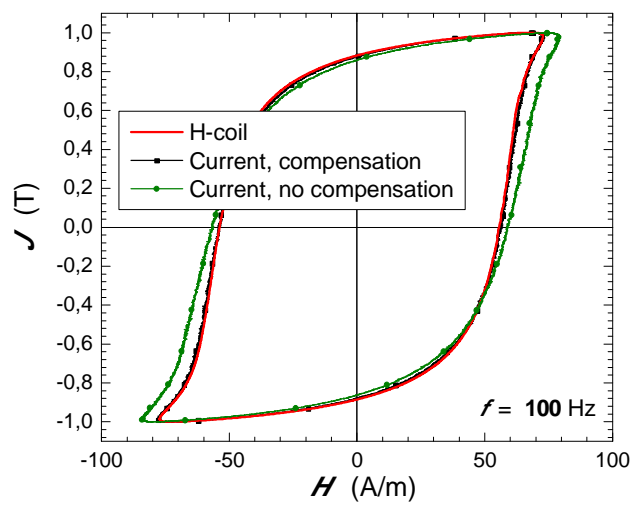

Fig. 7. NO Fe-Si sheets. $J_{\mathrm{p}}=1 \mathrm{~T}$. Comparison of the hysteresis loops measured by the $H$-coil method and by the current measurement method, with and without compensation.

It appears that the results by the $H$-coil method and the current method with compensation and wedge-shaped iron poles on the upper yoke show remarkable agreement. On the other hand, because of the additional loss contribution by the yokes, overestimated figures are obtained by use of the uncompensated permeameter, the higher $J_{\mathrm{p}}$ the higher the loss deviation (up to about $7 \%$ ). If the compensation procedure is applied to the standard permeameter configuration without wedge-shaped poles, the loss value is still overestimated (from $2 \%$ to $4 \%$ ), because the magnetic path cannot be fully 
198 constrained to the sample length $L_{\mathrm{i}}$ and is slightly longer. 231

199 The good agreement between the $H$-coil and the compensatea 2

200 current methods is confirmed by the corresponding 433

201 measured hysteresis loops shown in Fig. 7.

234

\section{B. HiB grain-oriented sheets}

Measurements of high permeability GO materials arê 204 demanding, because the MMF drop in the yokes may be far 205 from negligible with respect to the one in the sample. At the 206 same time, the localized measurements using the $H$-coil are 39 207 difficult, especially at low frequencies, because the signal can 208 be very small. This adds to the interest for the here proposed 209 solution, which includes also the measurement of the apparent 2 210 power. Fig. 8 compares the energy loss values obtained with 3 211 the $H$-coil method and the magnetizing current method, with 212 and without compensation, in the GO sheet. It is apparent that 245 213 the compensated permeameter and the $H$-coil measurements 6 214 provide close results. Under uncompensated conditions, one 7 215 finds instead that the current method overestimates the 216 magnetic loss by a substantial extent, especially at low 217 inductions (about $10 \%$ for $J_{\mathrm{p}}=0.5 \mathrm{~T}, 6 \%$ for $J_{\mathrm{p}}=1.5 \mathrm{~T}$ ). This 218 is expected, because the loss in the yoke depends only on 249 219 not on the type of material under test, and its ratio to the 1

220 sample loss increases with better materials

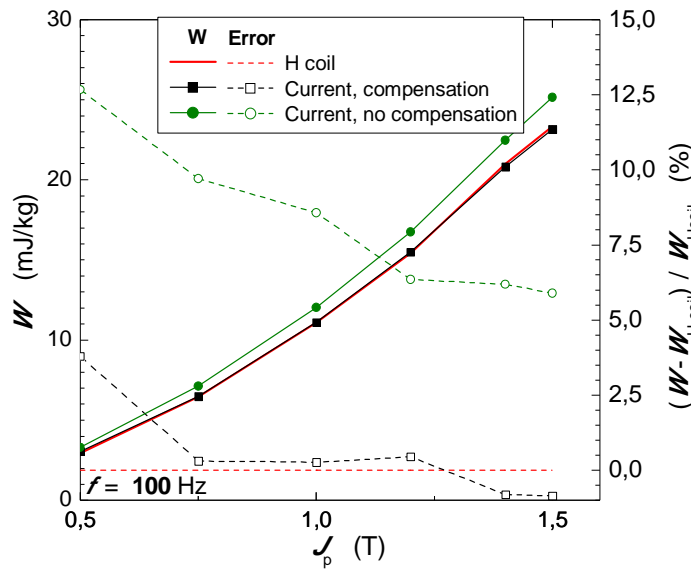
measured by the $H$-coil method and the current measurement method, with 3 and without compensation.

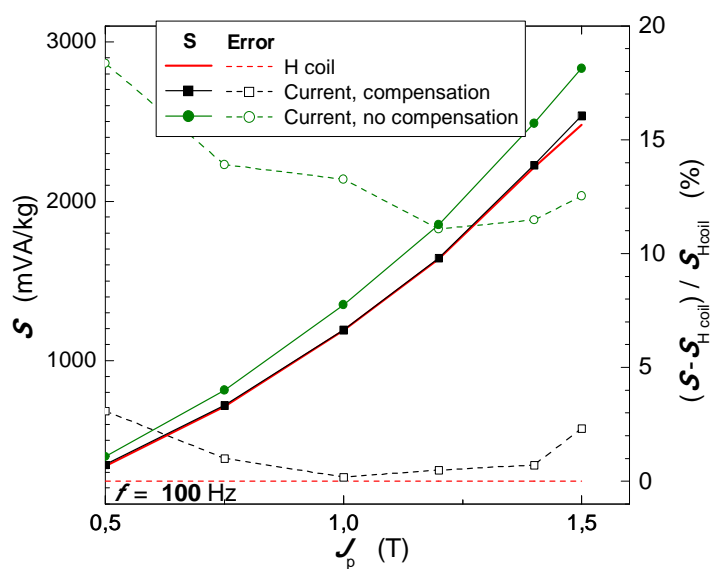

Fig. 9 shows the behavior of the measured apparent pow 295 Again, we find good agreement between the results provide97 the $H$-coil and compensated current measurements could be attributed to the difficulties intrinsic to the $H$-coil method (small signal, integration problems...). This further stresses the merits of the here proposed approach.

\section{CONCLUSIONS}

A permeameter has been developed, which applies a simple and effective magnetomotive force compensation method for the accurate characterization of soft magnetic steel sheets. It does not require specific sensors, except wedge shaped pole faces for the precise definition of the magnetic path length. It works on the principle of using the flux-closing yoke itself for both sensing and compensation.

The performances of this permeameter have been validated by measurements on non-oriented and grain-oriented $\mathrm{Fe}-\mathrm{Si}$ sheets, whose results excellently compare with the results provided by measurements performed using the tangential $H$ coil method.

\section{REFERENCES}

[1] W. H. Kim et al., "NE-Map-Based Design of an IPMSM for Traction in an EV," IEEE Transactions on Magnetics, vol. 50, no. 1, pp. 1-4, 2014.

[2] W. Hua, G. Zhang, and M. Cheng, "Analysis of Two Novel Five-Phase Hybrid-Excitation Flux-Switching Machines for Electric Vehicles," IEEE Transactions on Magnetics, vol. 50, no. 11, pp. 1-5, 2014.

[3] J. H. Lee and B. I. Kwon, "Optimal rotor shape design of a concentrated flux IPM-type motor for improving efficiency and operation range," IEEE Transactions on Magnetics, vol. 49, no. 5, pp. 2205-2208, 2013.

[4] J. Pippuri, A. Manninen, J. Keranen, and K. Tammi, "Torque density of radial, axial and transverse flux permanent magnet machine topologies," IEEE Transactions on Magnetics, vol. 49, no. 5, pp. 23392342, 2013.

[5] M. J. Kim et al., "Torque density elevation in concentrated winding interior PM synchronous motor with minimized magnet volume," IEEE Transactions on Magnetics, vol. 49, no. 7, pp. 3334-3337, 2013.

[6] L.K. Rodrigues and G.W. Jewell, "Model Specific Characterization of Soft Magnetic Materials for Core Loss Prediction in Electrical Machines," IEEE Transactions on Magnetics, vol. 50, no. 11, 2014.

[7] E. Barbisio, F. Fiorillo, and C. Ragusa, "Predicting Loss in Magnetic Steels Under Arbitrary Induction Waveform and With Minor Hysteresis Loops," IEEE Transactions on Magnetics, vol. 40, no. 4, pp. 1810$1819,2004$.

[8] IEC Standard Publication 60404-2, Part 2: Methods of measurement of the magnetic properties of electrical steel strip and sheet by means of an Epstein frame, 1996, Geneva, IEC Central Office.

[9] J Sievert, H. Ahlers, F. Fiorillo, L. Rocchino, M. Hall, and L. Henderson, "Magnetic measurements on electrical steels using Epstein and SST methods", PTB-Bericht, vol. E-74, pp. 1-28 (2001).

[10] J. Sievert, "Determination of ac magnetic power loss of electrical steel sheet: present status and trends," IEEE Transactions on Magnetics, vol. 20, no. 5, pp. 1702-1705, 1984 .

[11] F. Fiorillo, Measurement and characterization of magnetic materials.: North-Holland, 2004, p.286.

[12] R.S. Girgis, K. Gramm, J. Sievert, and M.G. Wickramasekara, "The single sheet tester. Its acceptance, reproducibility, and application issues on grain-oriented steel," Le Journal de Physique IV, vol. 8, no. PR2, pp. 729-732, 1998.

[13] IEC Standard Publication 60404-3, Part 3: Methods of measurement of the magnetic properties of electrical steel strip and sheet by means of a single sheet tester, 1992, Geneva, IEC Central Office.

[14] C. Appino et al., "International comparison on SST and Epstein measurements in grain-oriented $\mathrm{Fe}-\mathrm{Si}$ sheet steel," International Journal of Applied Electromagnetics and Mechanics, vol. 48, no. 2,3, pp. 123-133, 2015.

[15] J. Sievert et al., "New Data on the Epstein to Single Sheet Tester Relationship," Przeglad Elektrotechniczny, vol. 7, no. 13, pp. 1-3, 2013.

[16] A. Nafalski, A.J. Moses, T. Meydan, and M.M. Abousetta, "Loss measurements on amorphous materials using a field-compensated single-strip tester," IEEE Transactions on Magnetics, vol. 25, no. 5, pp. 4287-4291, 1989. 